\title{
Study on optical measurement technology for automotive covering parts
}

\author{
Xinhe Liang ${ }^{1, a^{*}}$ \\ ${ }^{1}$ school of material science and engineering, Henan university of science and \\ technology,471023,Luoyang, China \\ alxh8330@163.com
}

Keywords: Optical measurement, photogrammetry, point cloud, ICP method.

Abstract. Aim to demand for automobile covering parts of measurement, this paper puts forward a new kind of optical inspection method, which combines photography with dense point scanning measurement. In order to meet the requirement of measuring the covering parts of automobiles, this paper proposes a new kind of optical inspection method which combines photography with dense points scanning measurement. The photogrammetry is used for landmark coordinates measurement, dense point clouds are gathered by scanning measuring equipment, According to the distance between the landmark invariance, a serial of point clouds are coarse registered with public landmark, a improved ICP method is used to promote the accuracy of registration, Experiments show that ultimately discrete points model can realize optical measurement for automobile covering parts, measurement accuracy in the range of $0.1 \mathrm{~mm}$, it is satisfied the requirement of rapid optical measurement for automobile covering parts.

\section{Introduction}

Automotive covering part has the characteristics of large size, complex structure and complicated shape of the surface. In order to ensure the production quality, it is demanded to inspect fully surface. At present, the main measurement method is contact measurement. Three coordinate measuring is a typical contact measurement with high precision and low efficiency. With the rapid development of optical measurement technology, optical measurement should be adopted for covering parts of cars. Typical optical measurement systems are Germany GOM's ATOS and TRITOP products, XJTUDP, XJTUOM system, etc, these measurement precision are up to the $1 / 60000$, all can be quickly acquired surface data to be measured. Compared with the contact measurement method, optical measurement technology has the characteristics of less equipment investment, convenient and quick, and can meet the needs of the stamping parts field measurement [1].

Key technologies of optical measurement including mark point Photogrammetry, point cloud acquisition, point cloud registration, measurement error calculation and display etc.

\section{Mark point photogrammetry}

Before measurement, the code landmark and noncoding landmark are pasted on the surface of testing part, The precision scale is placed in the scene, and then the high precision digital camera is used to get a certain number of photos surrounding the artifacts from a different angle and position, mark point 3D coordinate is carry out through photo analysis by XJTUDP.

\section{Point cloud acquisition}

XJTUOM is a point cloud scanning equipment, it project the multi-frequency sinusoidal image on the surface of testing parts, binocular CCD camera captures a series of digital image, software system processes images to get the phase angle for every pixel, according to the phase angle coding and coplanar equation to determine the corresponding relationship of binocular camera image, and then get the depth of the image object [2]. Complex object surface usually need scan many times from different perspectives and multi point clouds are achieved. 


\section{Point cloud coarse registration}

In order to meet the requirements of profile measurement, point clouds which captured from different views and mark points which achieved with photography system XJTUOM need to be registered under the same coordinate system. First, XJTUOM identify mark points in images which captured by its binocular camera, second, mark points coordinate is calculated and distance set between difference mark points is carried out. According to the distance between the same landmark invariance, comparing the distance between difference mark points which captured with XJTUOM and the distance between mark points which carried out by XJTUDP, find the same landmark. This process is called point cloud coarse registration [3]. The key technology lies in the same mark point matching and finding.

Suppose there are two point set A and B which has public subset, and A is full field mark point set by photogrammetry. B is mark point set captured by a scan for local landmark with point cloud acquisition system. A and B exists at least three public mark points which don't in the collinear.

In point set $B$ choose three points $\left\{\mathrm{q}_{1}, \mathrm{q}_{2}, \mathrm{q}_{3}\right\}$, Distance is calculated by Eq. 1

$$
\mathrm{DB} 1=\left\|\mathrm{q}_{1}-\mathrm{q}_{2}\right\|, \mathrm{DB} 2=\left\|\mathrm{q}_{1}-\mathrm{q}_{3}\right\|, \mathrm{DB} 3=\left\|\mathrm{q}_{2}-\mathrm{q}_{3}\right\|
$$

Choose three points in point set $\mathrm{A}\left\{\mathrm{p}_{1}, \mathrm{p}_{2}, \mathrm{p}_{3}\right\}$, Distance is calculated by Eq. 2 .

$$
\mathrm{DA} 1=\left\|\mathrm{p}_{1}-\mathrm{p}_{2}\right\|, \mathrm{DA} 2=\left\|\mathrm{p}_{1}-\mathrm{p}_{3}\right\|, \mathrm{DA} 3=\left\|\mathrm{p}_{2}-\mathrm{p}_{3}\right\|
$$

Compare with DB1, DB2, DB3 and DA1, DA2, DA3, If they are equal respectively, then $\left\{\mathrm{q}_{1} 、 \mathrm{q}_{2}\right.$ 、 $\left.\mathrm{q}_{3}\right\}$ have the assured correspond relationship with $\left\{\mathrm{p}_{1}, \mathrm{p}_{2}, \mathrm{p}_{3}\right\}$. Transformation matrix is calculated using the corresponding point pairs, the point cloud can be registered by transformation matrix.

\section{Point cloud fine registration technology}

Registered multi point clouds describe the complete physical model. Under the influence of mark point recognition accuracy, however, registered point cloud data still has larger registration error, need to improve registration accurate.

ICP is a commonly used method for point cloud registration [4]. But the traditional ICP method is easily affected by the initial position, easy to fall into local optimum. In this paper, the traditional ICP method on the basis of accurate registration method is improved.

Introduce of ICP method. Suppose there are point set $\mathrm{P}=\left\{\mathrm{p}_{\mathrm{i}} \mid \mathrm{p}_{\mathrm{i}} \in \mathrm{R}^{3}, \mathrm{i}=1,2, \ldots, \mathrm{N}\right\}$, Reference point set $\mathrm{Q}=\left\{\mathrm{q}_{\mathrm{j}} \mid \mathrm{q}_{\mathrm{j}} \in \mathrm{R}^{3}, \mathrm{j}=1,2, \ldots, \mathrm{M}\right\}$, The steps of algorithm is

1) Set a threshold value $T>0$

2 ) For each point $p_{i}$ in the target point set $P$, search the nearest corresponding point $q_{j}$ from the reference point set $\mathrm{Q}$

3 ) To minimize the objective function to obtain registration parameters by Eq 3.

$\left\{R_{k}, T_{k}\right\}=\arg \min \sum_{i=1}^{n}\left\|\left(R_{k} p_{i}+T_{k}\right)-q_{i}\right\|^{2}$

4 ) Update the target point set $P, P_{k}{ }^{\prime}=R_{k} p_{i}+T_{k}$

5 ) Calculate the registration error between 2 iterations by Eq 4.

$d_{k+1}=\frac{1}{N} \sum_{i=1}^{N}\left\|P_{k+1}^{\prime}-P_{k}^{\prime}\right\|^{2}$ 
If $\left|d_{k+1}-d_{k}\right| \leq T$, Stop the iteration, Otherwise, return to step 2 to continue iterations.

\section{Improved ICP method.}

1 ) Point cloud sampling again

The precision of the ICP algorithm mainly depends on the accuracy of the matching point pairs, therefore, appropriate to reduce the amount of data that does not significantly impact on the accuracy of algorithm. This paper uses the uniform random sampling in the reference point set for the closest point. Sampling rate is $30 \%$. Closest point search process has high time complexity in the algorithm, sampling can reduce the number of the closest point for searching, significantly improve the efficiency of the algorithm.

2 ) Searching the matching point pairs

The closest point searching method may not be able to find truly corresponding points, affect accuracy of algorithm. Therefore, the similarity evaluation function is used to find the real match point in the adjacent points. For the target point set arbitrary point in the $\mathrm{P}$, seeking $\mathrm{k}$ the nearest points in reference point cloud Q. Then, then every point is evaluated by the similarity function. Evaluation function is Eq5.

$$
\operatorname{Sim}\left(\mathbf{p}_{i}, \mathbf{q}_{i}\right)=\left(\mathbf{n}_{p i} \cdot \mathbf{n}_{q i}\right) \exp \left(-\left\|\mathbf{p}_{i}-\mathbf{q}_{i}\right\|^{2} / h^{2}\right), \operatorname{Sim}\left(\mathbf{p}_{i}, \mathbf{q}_{i}\right) \geq \mu
$$

Here, $\mathrm{n}_{\mathrm{pi}}, \mathrm{n}_{\mathrm{qi}}$ is normal vector of points $\mathrm{p}_{\mathrm{i}}, \mathrm{q}_{\mathrm{i}}$ respectively. Truly matching points is the points which owned the biggest similarity value in $\mathrm{k}$ neighbor points. The distance and normal vector are two factors for similarity function, it increase with the similarly between the normal vector and increase with the decrease of the distance between points, when the similarity function is less than the given threshold, the decision that reference point is decided the wrong matching point pairs. The use of the similarity function can eliminate the wrong matching point pairs, make the point cloud registration more reliable.



Figure 1. A testing part

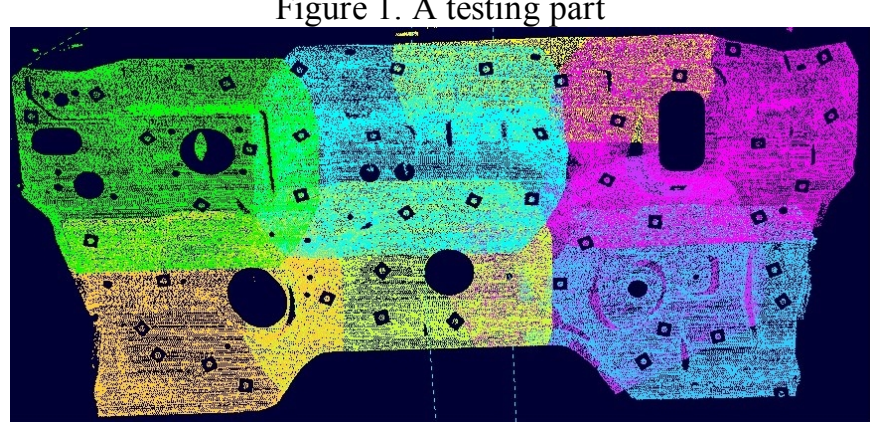

Figure 2 .Multi registered point clouds 


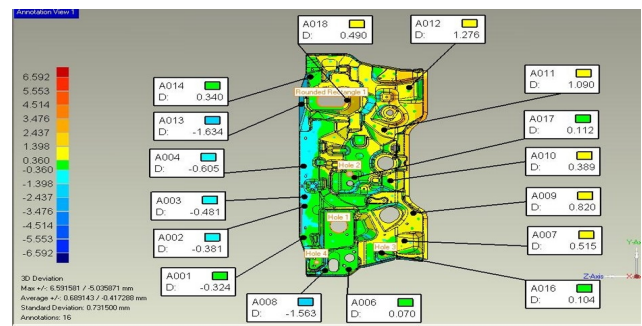

Figure 3.The results of the comparison the theoretical model with point cloud model.

\section{Experimental Verification}

A particular stampings part is tested for optical measurement. Figure 1 shows a testing part. Figure 2 shows multi registered point clouds, here, take $\mathrm{k}=3, \mu=0.6, \mathrm{~h}=3 \mathrm{~d}$ in improved ICP method, and $\mathrm{d}$ is average space in point cloud. Figure 3 shows the results of the comparison the theoretical model with point cloud model.

\section{Conclusion}

In this paper, a complete optical measurement method for automotive covering parts is proposed. Compared with the traditional contact measuring method, the new method has efficiency, low cost, and intuitive results. Detection accuracy can reach $0.1 \mathrm{~mm}$, this method can be used to meet the needs of stamping products measurement.

\section{References}

[1] Tao Jin, Yan Shan, Shui-guang Tong: submitted to Journal of computer aid design \&computer graphics 2001. in Chinses

[2] Lei Feng, Kangning Chen: submitted to Journal of XI'AN Jiaotong university 2002. in Chinses

[3] Xin Wang, Mingming Zhang, Xiao Yu: submitted to Optics and procision engineering 2012, in Chinses

[4] Blais G, Levine MD: submitted to IEEE Transactions on Pattern Analysis andMachine Intelligence . 1995 